\title{
Appearance of itinerant electrons detected by IR spectroscopy and its correlation with surface magnetism in Co-doped $\mathrm{TiO}_{2}$ nanopowders
}

\author{
E.V. Mostovshchikova ${ }^{1 *}$ A.Ye. Yermakov ${ }^{1,2}$, M.A. Uimin ${ }^{1,2}$, and A.S. Minin ${ }^{1,2}$ \\ ${ }^{1}$ M.N. Miheev Institute of Metal Physics of Ural Branch of Russian Academy of Sciences, 620108 Ekaterinburg, Russian Federation \\ ${ }^{2}$ Ural Federal University, 620083, Ekaterinburg, Russian Federation
}

\begin{abstract}
IR absorption and magnetic data for the titanium dioxide nanopowders are studied. Additional absorption in IR range is revealed in nanocrystalline $\mathrm{TiO}_{2}$ and Co-doped $\mathrm{TiO}_{2}$ after reducing annealing and explained by appearance of the oxygen vacancies and delocalised charge carriers. Increasing of the absorption in IR range after annealing of Co-doped $\mathrm{TiO}_{2}$ in vacuum (or decreasing of this contribution after annealing in air) correlates with arising (or vanishing) of ferromagnetic contribution.
\end{abstract}

\section{Introduction}

Titanium dioxide in nanostate is one of the intensively studied materials for nanotechnology applications in photocatalysis and photoelectrochemical solar cells, in sensors, spintronics, and medicine [1]. Various reducing treatments, creation of the oxygen vacancies, doping by $3 \mathrm{~d}$ ions modifies the electronic and magnetic properties of nano- $\mathrm{TiO}_{2}$ and this is the way for improvement of the properties which are important for practice.

Recently it was shown that doping $\mathrm{TiO}_{2}$ with Co-ions up to $4 \%$ and subsequent annealing in vacuum at $1000 \mathrm{~K}$ results in appearance of spontaneous magnetization at room temperature that was associated with the increase in the Co atoms content on the surface of nanoparticles [2]. The results were interpreted within the framework of core-shell model. It was assumed that the ferromagnetic ordering is a result of the interaction of delocalized electrons near the vacancies and the nearest neighbour cobalt atoms due to itinerant type interaction.

One of the methods that can answer the question about the existence of the delocalized states in semiconductor is the infrared spectroscopy and study of the absorption spectra in the "transparency window" between the fundamental and phonon absorption where delocalised charge carriers can manifest themselves. In the present work we try to establish the relation between the evolution of the magnetic properties and IR spectra in Co-doped $\mathrm{TiO}_{2}$ nanopowders under the reducing treatment as well. To separate influence of the Codoping and different treatments on the evolution of the IR spectra of the titanium dioxide the absorption data of undoped nanocrystalline $\mathrm{TiO}_{2}$ were studied also.

\section{Experimental details}

\footnotetext{
Corresponding author: mostovsikova@imp.uran.ru
}

Nanocrystalline $\mathrm{TiO}_{2}$ and $\mathrm{TiO}_{2}: \mathrm{Co}(\mathrm{Co}$ content is 1.5 at $\%$ ) were prepared by the hydrothermal method [2]. XRD indicates the formation of anatase structure. The size of the nanoparticles determined by TEM is about $60-80 \mathrm{~nm}$ in length and $20-25 \mathrm{~nm}$ in width.

Before any measurements and annealing the treatment of the synthesized $\mathrm{TiO}_{2}$ and $\mathrm{TiO}_{2}: \mathrm{Co}$ nanopowders in $\mathrm{HCl}$ was carried out to remove contamination on the surface, if any. These powders are denoted as the nanopowders in initial state. Then the reducing annealing was carried out in vacuum of $10^{-4} \mathrm{~Pa}$ at $\mathrm{T} \sim 1000 \mathrm{~K}$ during 30 minutes. Further, one part of the vacuum treated nanopowders was annealed in air at 670 $\mathrm{K}$ during 2 hours and another part was again etched in $\mathrm{HCl}$.

Magnetization of the nanocrystalline $\mathrm{TiO}_{2}$ :Co was measured at room temperature on the Faraday balance in the fields up to $12 \mathrm{kOe}$.

For the optical measurements nanopowders of $\mathrm{TiO}_{2}$ or $\mathrm{TiO}_{2}$ :Co were compacted with CsI under the pressure $\mathrm{P}=5 \mathrm{GPa}$.To study the evolution of the IR absorption of $\mathrm{TiO}_{2}$ or $\mathrm{TiO}_{2}$ :Co nanopowders the transmission of the obtained composites in the range $0.9-12.5 \mu \mathrm{m}$ was measured and optical density was calculated as $\mathrm{D}(\lambda)=\ln (1 / \mathrm{T}(\lambda))$. The transmission spectrum of CsI was taken into account.

\section{Results and discussion}

Figure 1 demonstrates the magnetization curves of the nanocrystalline $\mathrm{TiO}_{2}: \mathrm{Co}$. It is shown that initial $\mathrm{TiO}_{2}: \mathrm{Co}$ nanopowder is paramagnetic. After annealing of the nanopowder in vacuum behaviour of the magnetization curve changes drastically, and the ferromagnetic contribution is clearly observed. Saturation magnetization $\mathrm{M}_{\mathrm{s}}$ at $\mathrm{T}=300 \mathrm{~K}$ is about $0.85 \mathrm{emu} / \mathrm{g}$. Subsequent annealing of the $\mathrm{TiO}_{2}$ :Co powder in air 
results in decreasing of the magnetization, but $\mathrm{M}(\mathrm{H})$ curve still demonstrates ferromagnetic-like character: the saturation of $\mathrm{M}$ is observed, the value of $\mathrm{M}_{\mathrm{s}}$ is about 0.04 $\mathrm{emu} / \mathrm{g}$ (the insert in Figure 1).

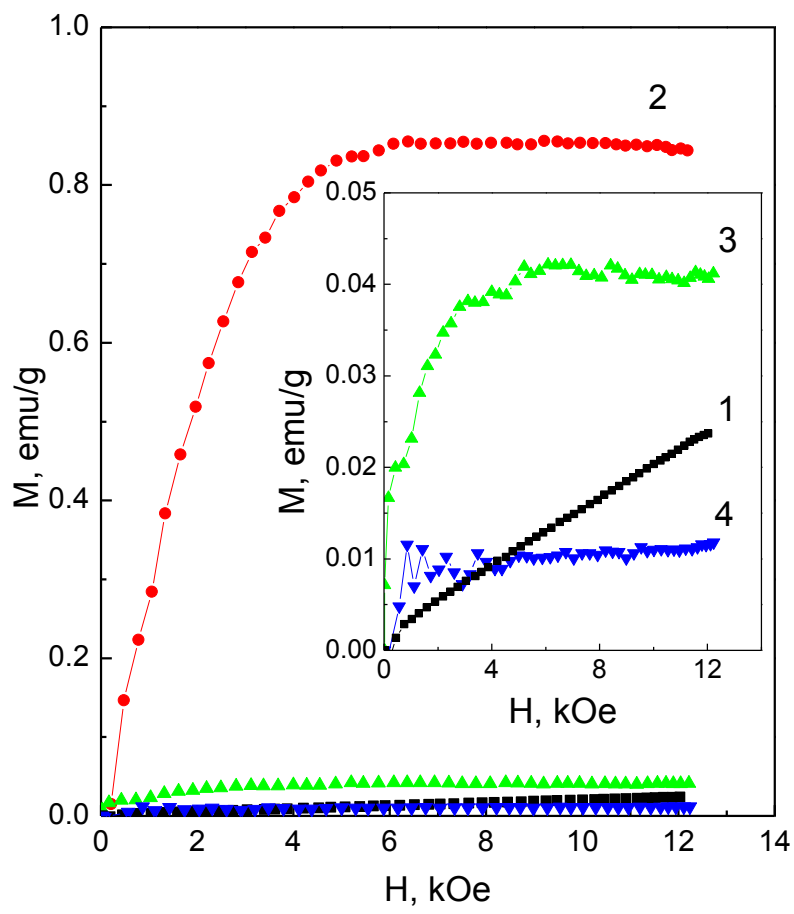

Fig. 1. The magnetization curves for $\mathrm{TiO}_{2}$ :Co nanopowders. 1 - initial state, 2 - annealing in vacuum, 3 - annealing in vacuum and further annealing in air, 4 - annealing in vacuum and etching in $\mathrm{HCl}$.

$\mathrm{HCl}$-etching $\mathrm{TiO}_{2}$ :Co nanopowder annealed in vacuum affects magnetization more significantly then the annealing in air. The curve $\mathrm{M}(\mathrm{H})$ becomes close to that of initial powder $\mathrm{TiO}_{2}$ :Co. Disappearing of the magnetization after etching in $\mathrm{HCl}$ is in agreement with the conclusion made in [2] that there is the tendency of
Co-ions to localise on the surface and subsurface layers resulting in increase in the Co atoms content there as compared with that in the bulk.

The optical density spectra $\mathrm{D}(\lambda)$ of $\mathrm{TiO}_{2}: \mathrm{Co}$ nanopowders in comparison with that of undoped $\mathrm{TiO}_{2}$ are presented in Figure 2. $\mathrm{D}(\lambda)$ curves for the powders with and without Co-ions have the same character. The increase of the absorption in the long-wave range of the spectra is associated with the phonon band with the frequency $v_{\mathrm{LO}}=876 \mathrm{~cm}^{-1}(\lambda=11.41 \mu \mathrm{m})$ [3]. The increase of the absorption in the shortwave range of the spectra is caused by the presence of the band at $\lambda \sim 1 \mu \mathrm{m}(\mathrm{E} \sim 1.2$ $\mathrm{eV}$ ) associated with the polaron optical absorption due to self-trapped electrons undergoing hopping between $\mathrm{Ti}^{4+}$ and $\mathrm{Ti}^{3+}[4]$. In $\mathrm{D}(\lambda)$ spectra of the powders in the initial state there are the weak absorption bands at $\lambda=7.3 \mu \mathrm{m}$, $6.2 \mu \mathrm{m}$, and $3.1 \mu \mathrm{m}$ which correspond to the vibrations of b- $\mathrm{CO}_{3}$, water, and $\mathrm{O}-\mathrm{H}$ strongly adsorbed on the nanoparticles surface [5]. Doping with Co does not influence the spectra at $\lambda>4 \mu \mathrm{m}$ and results in the increase of the absorption at $\lambda<4 \mu \mathrm{m}$ that is caused by the appearance of the absorption bands attributed to the d-d- transitions in Co-ions [6].

After annealing of nanopowders in vacuum, the following evolution of the optical spectra occurs. The bands at $\lambda=7.3 \mu \mathrm{m}, 6.2 \mu \mathrm{m}$, and $3.1 \mu \mathrm{m}$ are almost disappeared, that can be explained by removal of the corresponding molecules from the nanoparticles surface. The absorption increases both for undoped and Codoped titanium dioxide (curves 2 in Fig.2). The growth of the absorption in the short-wave range is the result of the appearance additional absorption band at $\sim 0.8 \mu \mathrm{m}$ attributed to $\mathrm{Ti}^{3+}$ ions [8].

The more intriguing change of $D(\lambda)$ spectra is observed near phonon bands. The increase of the absorption near phonon edge begins at shorter wavelength (at $\lambda \sim 7 \mu \mathrm{m}$ ) and $\mathrm{D}(\lambda)$ curves in the range $\lambda>7 \mu \mathrm{m}$ is turned up in contrast to the curves of the powders in initial state, that is typical for materials with delocalised charge carriers. It is known that the higher
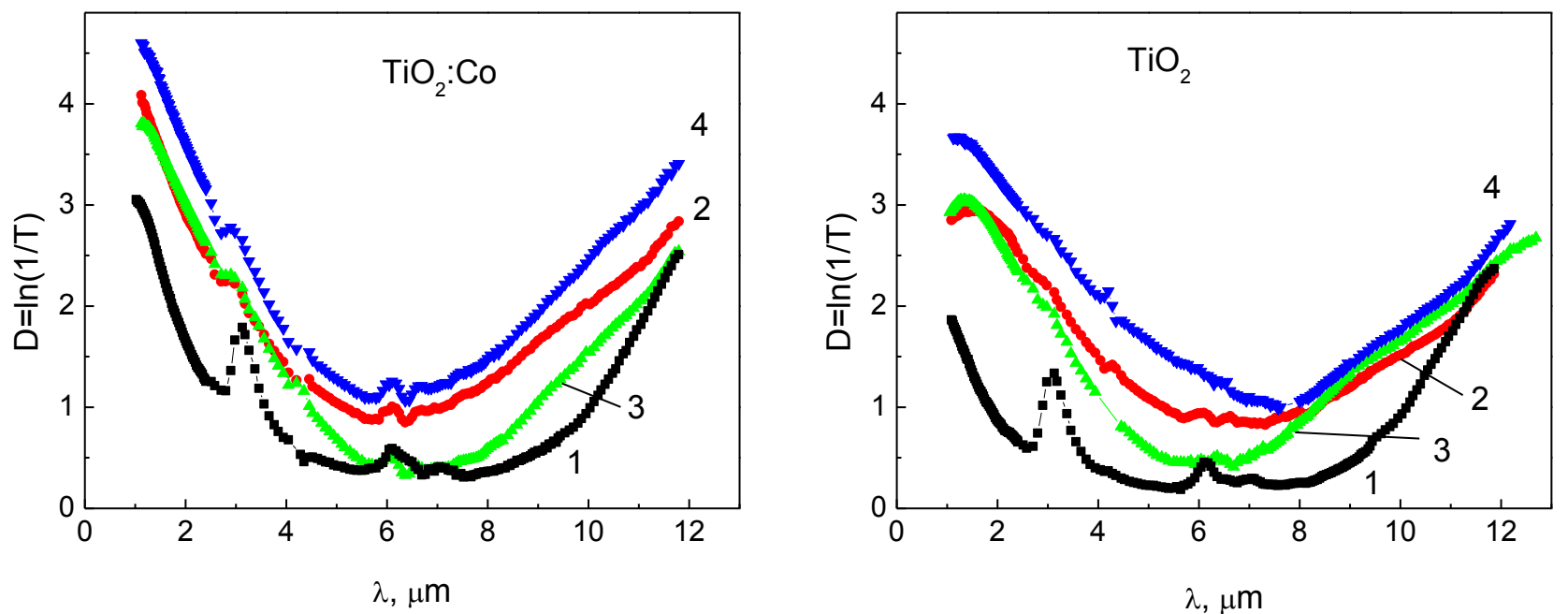

Fig. 2. Optical density spectra of $\mathrm{TiO}_{2}: \mathrm{Co}(\mathrm{a})$ and $\mathrm{TiO}_{2}$ (b) nanopowders. 1 - initial state, 2 - annealing in vacuum, 3 - annealing in vacuum and further annealing in air, 4 - annealing in vacuum and etching in $\mathrm{HCl}$ 
conductivity of the material corresponds to the higher absorption coefficient in IR range. It is important that delocalised charge carriers appears not only in $\mathrm{TiO}_{2}$ : $\mathrm{Co}$ powder but in undoped $\mathrm{TiO}_{2}$ too. Therefore, the origin of these carriers is not connected with the possible appearance of the droplets of metallic Co on the nanoparticles surface. So, the growth of the absorption and the appearance of Drude-like additional contribution in $\mathrm{D}(\lambda)$ spectra may be explained by appearance of delocalised charge carriers associated with the oxygen vacancies.

Annealing reduced $\mathrm{TiO}_{2}$ or $\mathrm{TiO}_{2}: \mathrm{Co}$ nanopowders in air results in decrease of the absorption (curves 3 in Fig.2), but some residual Drude-like contribution remains. It means that some amount oxygen vacancies still remains. Decrease of the absorption of nanopowders after annealing in air correlates with the decrease of the magnetization (Fig.1) and is in agreement with the hypothesis about itinerant type of magnetism [2].

As one can see, if nanopowder contains Co-ions, change of the absorption after the reducing or oxidizing annealing is somewhat stronger than that for undoped $\mathrm{TiO}_{2}$. One of the possible reasons is that doping $\mathrm{TiO}_{2}$ with cobalt is accompanied by a significant increase in the concentration of the oxygen vacancies, as it was shown by study of the electron paramagnetic resonance [2]. Another reason may be so that segregations of Coions on the surface of the nanopowder after annealing in vacuum may lead to some segregation of the delocalised charge carriers. As a result, their contribution into the optical spectra is stronger.

Now let us consider the influence of $\mathrm{HCl}$ etching $\mathrm{TiO}_{2}$ or $\mathrm{TiO}_{2}$ :Co nanopowders annealed in vacuum on the optical density spectra. Although the magnetization of $\mathrm{TiO}_{2}$ :Co after $\mathrm{HCl}$-etching decreases more strongly than after annealing in air, the absorption does not decreases (curves 4 in Fig.2) but even slightly increases. It again indicates that delocalised charge carriers do not appear from the metallic Co-droplets, if any. The distinction in the influence of HCl-treatment on the magnetic and optical properties may be explained by the difference of the thickness of surface layer of nanoparticles, in which the charge carriers connected with oxygen vacancies appear or the magnetization is formed.

\section{Conclusions}

We have reported the IR absorption data (optical density spectra $\mathrm{D}(\lambda)$ ) of nanocrystalline $\mathrm{TiO}_{2}$ : $\mathrm{Co}$ and $\mathrm{TiO}_{2}$ after different heat treatment and etching in $\mathrm{HCl}$.

In the optical density spectra of $\mathrm{TiO}_{2}$ and $\mathrm{TiO}_{2}: \mathrm{Co}$ nanopowders after annealing in vacuum an additional absorption of Drude-like character appears. It is revealed that there is the correlation between the appearance and disappearance of the ferromagnetic-like magnetization and Drude-like contribution after reducing or oxidizing annealing, respectively. It is shown that Drude-like contribution is not connected with possible forming of metallic Co-rich droplets. The results support the hypothesis about itinerant type of surface magnetism in Co-doped $\mathrm{TiO}_{2}$ nanopowders.

This work was supported by the Russian Scientific Foundation, Grant No. 16-12-10004.

\section{References}

1. X. Chen, Chin. J. Catal., 30, 839 (2009).

2. A.Ye. Yermakov, G.S. Zakharova, M.A. Uimin et al., J. Phys. Chem. C 120, 28857 (2016)

3. R. J. Gonzalez, R. Zallen, and H. Berger, Phys.Rev.B 55, 7014 (1997)

4. C. A. Triana, C. G. Granqvist, and G. A. Niklasson, J. Appl. Phys. 119, 015701 (2016)

5. C. Yan, W. Yi, H. Yuan, et al, Environmental Progress \& Sustainable Energy 33, 419 (2014)

6. B. Babu, Ch. Rama Krishna, Ch. Venkata Reddy et al., Spectrochimica Acta Part A: Molecular and Biomolecular Spectroscopy 109, 90 (2013)

7. V.N. Kuznetsov and N. Serpone, J. Phys. Chem. C, 113, 15110 (2009) 